\title{
Low frequency voltage clamp: recording of voltage transients at constant average command voltage
}

\author{
Florian Peters, Arne Gennerich, Dirk Czesnik, Detlev Schild* \\ Physiologisches Institut, Universität Göttingen, Humboldtallee 23, D 37073 Göttingen, Germany
}

Received 24 March 2000; received in revised form 12 April 2000; accepted 17 April 2000

\begin{abstract}
We implemented a simple feedback system that modifies the conventional current clamp mode of a patch clamp amplifier so that transient potentials, such as action potentials and synaptic potentials, can be measured as in the usual current clamp, while the average membrane potential is kept constant at a value chosen by the user. The circuit thus works like the current clamp for high frequency signals and like a voltage clamp for low frequency signals. We delineate its transfer properties and give application examples. (C) 2000 Elsevier Science B.V. All rights reserved.
\end{abstract}

Keywords: Voltage clamp; Current clamp; Patch clamp

\section{Introduction}

Recordings of action potentials (APs) or postsynaptic potentials are usually performed in the current clamp mode of the patch clamp technique (Hamill et al., 1981). In many situations it would be desirable to record APs or synaptic potentials at a selectable fixed average voltage. This would, for instance, be useful: (i) if the shape of APs depend on the voltage at which they are elicited; (ii) if spiking rate is to be measured as a function of the average membrane potential; (iii) if synaptic potentials are to be observed as a function of membrane voltage; or (iv) if the spiking rate must be increased in order to be able to observe inhibition of spiking.

Clearly, the classical patch clamp configurations do not allow recording voltage transients while holding the average voltage fixed. One would like an amplifier that works in the current clamp mode for fast signals and in the voltage clamp mode for slow signals.

In this paper, we describe a simple electronic circuit, which we name low frequency voltage clamp (IfVC). When added to a patch clamp amplifier, a new operat-

\footnotetext{
* Corresponding author. Tel.: +49-551-395915; fax: +49-551398399.

E-mail address: dschild@gwdg.de (D. Schild).
}

ing mode is achieved with the desired characteristics. Action, receptor or synaptic potentials are accurately recorded, while, on a longer time scale, the average membrane potential is kept constant.

\section{Experimental procedures}

\subsection{Circuitry of the low frequency voltage clamp}

Fig. 1A shows a schematic diagram of a cell membrane connected to the low frequency voltage clamp circuitry. The basic principle of this circuitry is to lowpass filter the membrane potential $u_{\mathrm{m}}$ (as measured in the current clamp), compare the resulting voltage $u_{\mathrm{f}}$ to a reference voltage $u_{\text {ref }}$, amplify the difference voltage $u_{\mathrm{ref}}-\mathrm{u}_{\mathrm{f}}$ by a factor $G=R_{4} / R_{3}$ and inject a current $I_{\text {inj }}=\left(\left(u_{\text {ref }}-u_{\mathrm{f}}\right) G-u_{\mathrm{m}}\right) / R_{\text {inj }}$ into the cell. Due to this feedback, the cell's membrane is thus clamped to the low pass filtered membrane potential. Hence, the name low frequency voltage clamp.

The above schematic diagram can be simplified in two respects. First, the time required for charging the cell (time constant $R_{\text {inj }} C_{\mathrm{m}}$ ) can be neglected, because it is usually in the order of $100 \mu$ s and thus, much faster than any other process in the circuit. We may therefore assume that the control amplifier acts instantaneously 
upon $u_{\mathrm{m}}$. Second, as the control amplifier clamps the voltage $u_{\mathrm{m}}$ only at low frequencies, it is convenient to think of $u_{\mathrm{m}}$ as the sum of an average voltage $\bar{u}_{\mathrm{m}}$ and a signal voltage $u_{\mathrm{s}}$. If $u_{\mathrm{s}}$ has only high frequency components, the amplifier will virtually not affect $\bar{u}_{\mathrm{m}}$. If $u_{\mathrm{s}}$ has low frequency components, there will be a negative feedback to keep $\bar{u}_{\mathrm{m}}$ at $u_{\text {ref }}$. With these simplifications the equivalent control circuit becomes very simple (Fig. 1B). Note that in this circuit, $u_{\mathrm{m}}$ is the membrane voltage of a real cell and it is assumed that this voltage is measured by a patch clamp amplifier in the current clamp mode and then fed to the feedback lowpass $R_{\mathrm{f}} C_{\mathrm{f}}$. The current signal $I_{\text {inj }}$ is added at the command summing point of the amplifier, where all currents to be injected are added (Fig. 1C). For those readers interested in building the circuit we include the full sche-

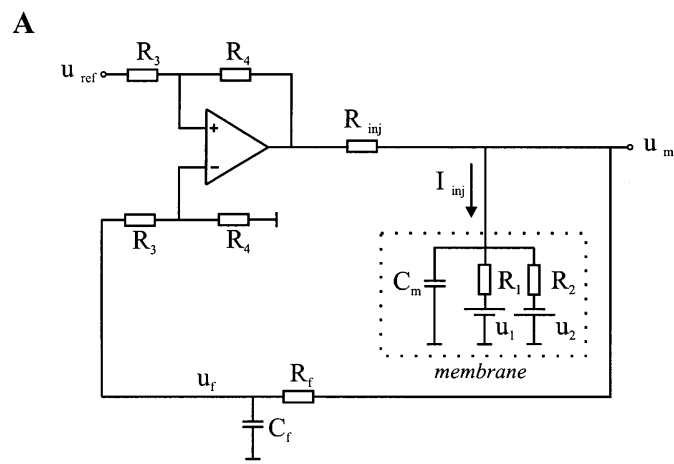

B

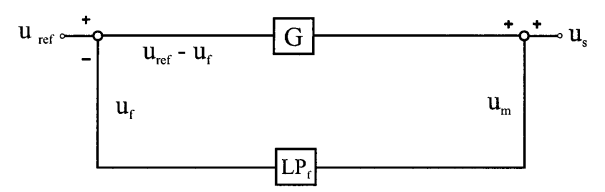

C

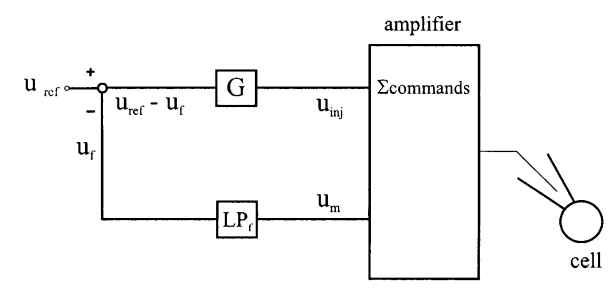

Fig. 1. Schematic electronic circuit of the low frequency voltage clamp. (A) The dotted box represents a cell membrane with a capacitance $C_{\mathrm{m}}$ and two conductances $\left(1 / R_{1}\right)$ and $\left(1 / R_{2}\right)$ with corresponding reversal potentials $u_{1}$ and $u_{2}$. The cell is embedded in the lfVC control circuit. $R_{\mathrm{f}}$, feedback resistance; $C_{\mathrm{f}}$, feedback capacitance; $u_{\mathrm{f}}$, lowpass filtered membrane voltage. By choosing various resistances $R_{\mathrm{f}}, \tau_{\mathrm{f}}$ was variable between 4.4 and $1020 \mathrm{~s} . R_{4} / R_{3}$ is a ratio of resistances determining the gain $G$ of the control amplifier. In this paper, $G=100 . u_{\text {ref }}$, reference voltage; $R_{\text {inj }}$, injection resistance $(2 \mathrm{k} \Omega$ in the EPC7); $u_{\mathrm{m}}$, membrane voltage. (B) Control circuit equivalent to part B. (C) lfVC attached to a patch clamp amplifier. The membrane voltage $u_{\mathrm{m}}$ is measured in the current clamp mode and the signal $G\left(u_{\text {ref }}-u_{\mathrm{f}}\right)$ is fed to the command summing point of the patch clamp amplifier. matic diagram (Fig. 2) which corresponds to the flow chart shown in the left half of Fig. 1C.

\subsection{Transfer behavior of the low frequency voltage clamp}

Assuming $u_{\mathrm{s}}=0$, the circuit's closed-loop transfer function $u_{\mathrm{m}} / u_{\text {ref }}$ is given by (Ogata, 1996)

$u_{\mathrm{m}} / u_{\mathrm{ref}}(\mathrm{f})=\frac{G H}{G+H}$

with $H=1+j 2 \pi \mathrm{f} \tau_{\mathrm{f}}$ and $\tau_{\mathrm{f}}=R_{\mathrm{f}} C_{\mathrm{f}} \cdot\left|u_{\mathrm{m}} / u_{\text {ref }}\right|(\mathrm{f})$ is shown in Fig. 3A. For a high gain and at low frequencies $\mathrm{f}$, the control ratio tends to unity, while at high frequencies it equals to the gain $G$. In our case $G=100$.

To obtain the system's response to a step $u_{\text {ref }}$, we apply the inverse Fourier transform to the function $u_{\mathrm{m}}(\mathrm{f})$,

$u_{m}=u_{r e f}(f) \cdot \frac{G H}{G+H}$,

which gives

$u_{\mathrm{m}}(t)=u_{\mathrm{ref}}\left\{\frac{G}{G+1}\left(1-\mathrm{e}^{-t / \tau}\right)+G \cdot \mathrm{e}^{-t / \tau}\right\}$

(Fig. 3B, dashed line), whereby $\tau=\tau_{\mathrm{f}} /(G+1)$. The first term describes $u_{\mathrm{m}}$ approaching the voltage $(G /(G+$ 1)) $u_{\text {ref }}$ (rather that $\left.u_{\text {ref }}\right)$, the asymptotic deviation $(1-$ $G /(G+1))$ being characteristic for $P$-type control systems. The second term in brackets, which is an exponential with amplitude $G u_{\text {ref }}$, is brought about by the abrupt increase in voltage at the beginning of the step. In order to avoid an overshoot of $u_{\mathrm{m}}$ while setting $u_{\text {ref }}$, we lowpass filter $\left(\tau_{\text {ref }}=4.4 \mathrm{~s}\right)$ the reference voltage prior to feeding it to the control circuit. Fig. 3B compares the responses to a step (truncated, exponential signal) and to a lowpass filtered step $\left(\tau_{\text {ref }}=4.4 \mathrm{~s}\right)$.

For a constant reference voltage, e.g. $u_{\text {ref }}=0$, the transfer function $u_{\mathrm{m}} / u_{\mathrm{s}}$ with respect to the cell's membrane signal $u_{\mathrm{s}}$ is given by (Ogata, 1996).

$u_{\mathrm{m}} / u_{\mathrm{s}}(f)=\frac{H}{G+H}$

For signals $u_{\mathrm{s}}(t)$ with high frequency components, $f \geq 1 / \tau_{\mathrm{f}}$, the transfer function tends to unity, i.e. $\left|u_{\mathrm{m}}\right| \sim$ $\left|u_{\mathrm{s}}\right|$. In contrast, low frequency signals $u_{\mathrm{s}}$ are attenuated by a factor of $(G+1)$, i.e. $u_{\mathrm{m}} \rightarrow u_{\mathrm{s}} /(G+1)$ for $\mathrm{f} \rightarrow 0$.

In the time domain, we give the system's response to a rectangular function, i.e. $u_{\mathrm{s}}=u_{\mathrm{o}}$ for $0<t<t_{1}$ and $u_{\mathrm{s}}=0$, otherwise (Fig. 3C). It is given by 


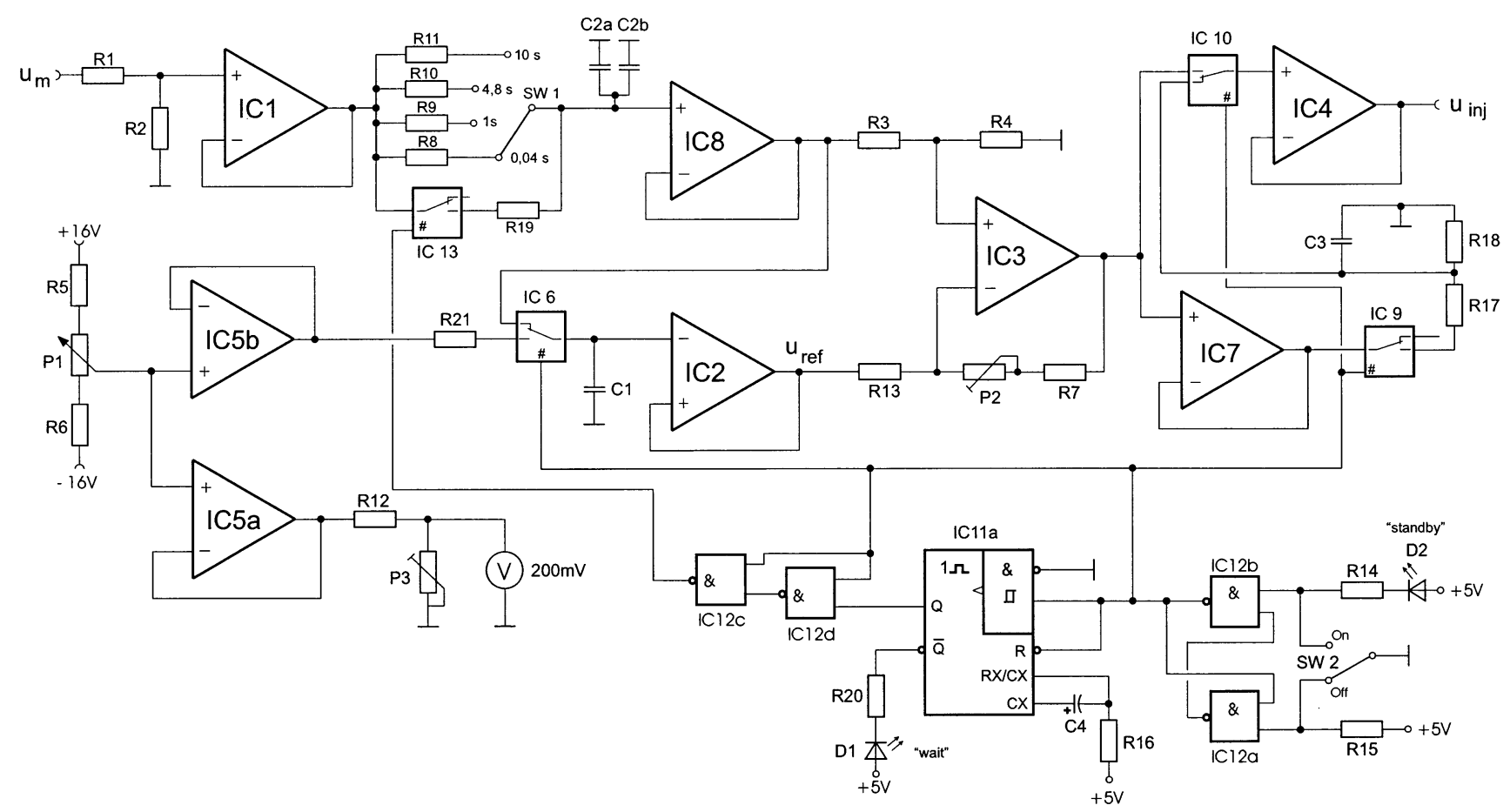

Fig. 2. Detailed schematic diagram of the low frequency voltage clamp. The reference voltage $u_{\text {ref }}$ is set using the potentiometer P1 shown on the left. The cell's membrane voltage, picked up in the patch clamp amplifier (in the EPC7, e.g. at the output of A106A) is fed to IC1 (upper left) and then lowpass filtered $\left(C_{2}\right.$ and e.g. $\left.R_{8}\right)$. IC3 is the control amplifier with a gain set to 100 . The injection voltage $u_{\text {inj }}$ is the output of the buffer IC4, which needs to be attached to the command summing point of the patch clamp amplifier via $R_{\text {inj }}$ (e.g. in the EPC7, via $2 \mathrm{k} \Omega$ to A106b). The rest of the circuit are analog switches, NANDs and a flip flop, which prevent the output $u_{\text {inj }}$ from overshooting when the circuit is switched on or off (SW2). IC1, 2, 3, 4, 7: OP27; IC8: OPA111; IC5: TL082; IC6, 9, 10, 13: TL607; IC11: 74LS221; IC12: 74LS00; D1, 2: LEDs (2 mA); C1, $\mathrm{C} 2$ a,b: $100 \mu \mathrm{F}$; C3: $1 \mu \mathrm{F}$; C4: $470 \mu \mathrm{F}$; R1, R17: $100 \Omega$; R2: $1 \mathrm{M} \Omega$; R3, R13, R19: $200 \Omega$; R4: $20 \mathrm{k} \Omega$; R5, R6: $51 \mathrm{k} \Omega ; \mathrm{R} 7: 18 \mathrm{k} \Omega$; R8: $22 \mathrm{k} \Omega$; R9: $500 \mathrm{k} \Omega$; R10: $2.4 \mathrm{M} \Omega$; R11: $5.1 \mathrm{M} \Omega ; \mathrm{R} 12: 10 \mathrm{k} \Omega ; \mathrm{R} 14, \mathrm{R} 15: 4.7 \mathrm{k} \Omega$; R16: $33 \mathrm{k} \Omega$; R18: 1.8 M $\Omega$; R20: $470 \Omega$; R21: $44 \mathrm{k} \Omega$. P1: $10 \mathrm{k} \Omega$; P2: $5 \mathrm{k} \Omega$; P3: $2 \mathrm{k} \Omega$.

$$
\begin{aligned}
u_{\mathrm{m}}(t)= & u_{\mathrm{o}}\left\{\frac{1}{G+1}\left(1-\mathrm{e}^{-t / \tau}\right)+\mathrm{e}^{-t / \tau}\right\} \\
& -\varepsilon\left(t-t_{1}\right) u_{\mathrm{o}}\left\{\frac{1}{G+1}\left(1-\mathrm{e}^{-\left(t-t_{1}\right) / \tau}+\mathrm{e}^{-\left(t-t_{1}\right) / \tau}\right)\right\}
\end{aligned}
$$

$\varepsilon\left(t-t_{1}\right)=1$ for $t>t_{1}$ and 0 , otherwise. Fig. 3D shows the responses $u_{\mathrm{m}}$ of the control circuit to a rectangular impulse $u_{\mathrm{s}}$ of $50 \mathrm{~ms}$ duration, whereby the responses are shown for three different feedback time constants $\tau_{\mathrm{f}}$. While for $\tau=4.8 \mathrm{~s}$, the response is virtually identical to the input $u_{\mathrm{s}}$, shorter time constants ( $\tau=1 \mathrm{~s}$ or $44 \mathrm{~ms}$ ) clearly lead to an exponential time course of the responses. For $u_{\mathrm{m}}$ to reflect $u_{\mathrm{s}}$, the control time constant has to be sufficiently large. Otherwise, a distortion of the signal envelope as well as an after effect of the control circuit have to be expected (see Fig. 3D for $t>50 \mathrm{~ms})$.

\subsection{Slice preparation}

Tadpoles of Xenopus laevis (stage 49-54) (Nieuwkoop and Faber, 1956) were anaesthetized and immobilized in a mixture of ice and water. A block of tissue containing the mucosae, the olfactory nerves and the anterior two thirds of the brain was cut out and kept in frog Ringer solution (solution B, Table 1). The tissue was glued on the stage of a vibroslicer (Model 752M, Campden Instruments Ltd., Longh Borough, UK) and cut horizontally into $150 \mu \mathrm{m}$ thick slices. Olfactory mucosae were also sectioned horizontally. The slices were placed under a harp-like grid in a recording chamber and viewed using the Nomarski optics of an Axioskop 2 (Zeiss, Göttingen, Germany). Olfactory receptor neurons were easily recognized by their characteristic shape, while mitral cells were chosen according to the layering of the olfactory bulb (Nezlin and Schild, 2000).

\subsection{Recording}

Patch electrodes, with a tip diameter of $1-2 \mu \mathrm{m}$ and $\approx 7-10 \mathrm{M} \Omega$, were fabricated from borosilicate glass (1.8 mm outer diameter; Hilgenberg, Malsfeld, Germany) using a two-stage electrode puller (Narishige, Tokyo). The pipettes were fire-polished. Pulse protocols, data acquisition and evaluation programs were written in ' $C$ '. Pulses were delivered from a microcon- 
troller (Schild et al., 1996) to a D/A converter and then to the patch-clamp amplifier (EPC7; List, Darmstadt, Germany). Currents and voltages were recorded on videotape using a PCM unit (Instrutech, Elmont, NY). The data were digitized off-line using an 8-pole Bessel filter, an A/D converter and a PC. Further data analysis was performed on a PC under LINUX.

\subsection{Solutions and stimulus application}

The compositions of the standard bath solution (B) and the intracellular solutions $\left(I_{1}, I_{2}\right)$ are listed in Table 1. All solutions were at $\mathrm{pH}$ 7.8. The osmo-

A

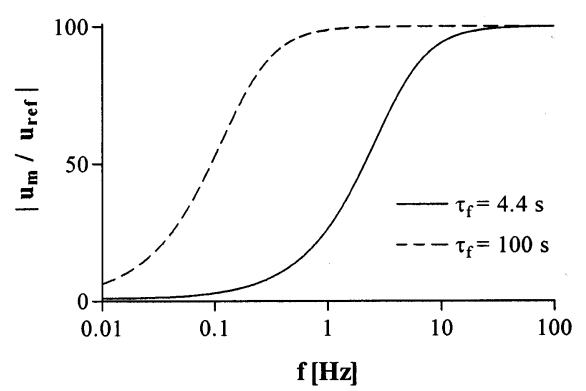

C

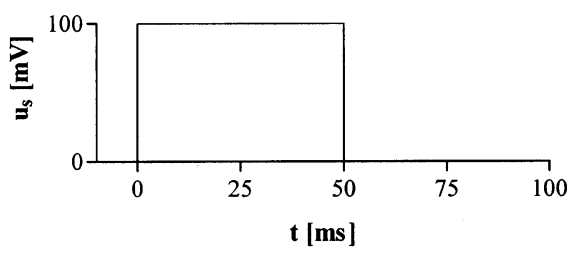

larities of the bath and the pipette solutions were adjusted to 230 and $190 \mathrm{mOsmol}$, respectively. For the odorants, we used an extract of amphibia food based on algae (Mikrozell, Dohse Aquaristik, Bonn, Germany) and dissolved in solution $B$. In the experiments on mitral cells, the chloride reversal potential was set to $-44.6 \mathrm{mV}$. The bath solution was applied by gravity feed from a storage syringe through a funnel drug applicator (Schild, 1985) to the recording chamber. The flow was $250 \mu \mathrm{l} / \mathrm{min}$. Odorants were pipetted directly to the funnel without stopping the flow. TTX and $\mathrm{Cd}^{2+}$ were dissolved in the bath solution.
B

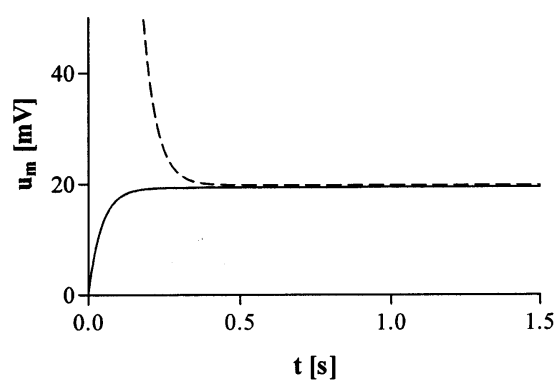

D

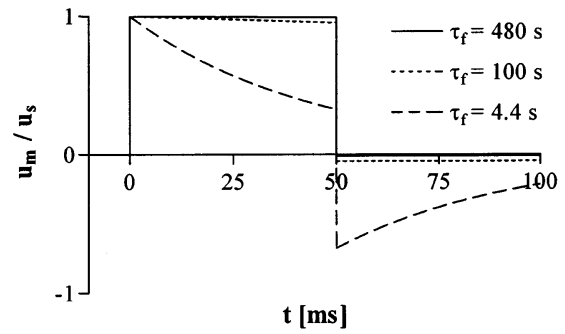

Fig. 3. Transfer and response functions of the lfVC. (A) Closed-loop transfer function of the circuit shown in Fig. $1 \mathrm{~B}$ for $\tau_{\mathrm{f}}=4.4 \mathrm{~s}$ and $\tau_{\mathrm{f}}=100$ s. (B) System response $u_{\mathrm{m}}(t)$ to a step in the reference voltage from 0 to $20 \mathrm{mV}$ (dashed line, truncated); the continuous line is the response to a lowpass filtered step from 0 to $20 \mathrm{mV}$, whereby the time constant of the first order lowpass was $4.4 \mathrm{~s}$. (C,D) System responses (D) to the box function shown in (C). For further explanation, see text.

Table 1

Ion composition of solutions used ${ }^{\mathrm{a}}$

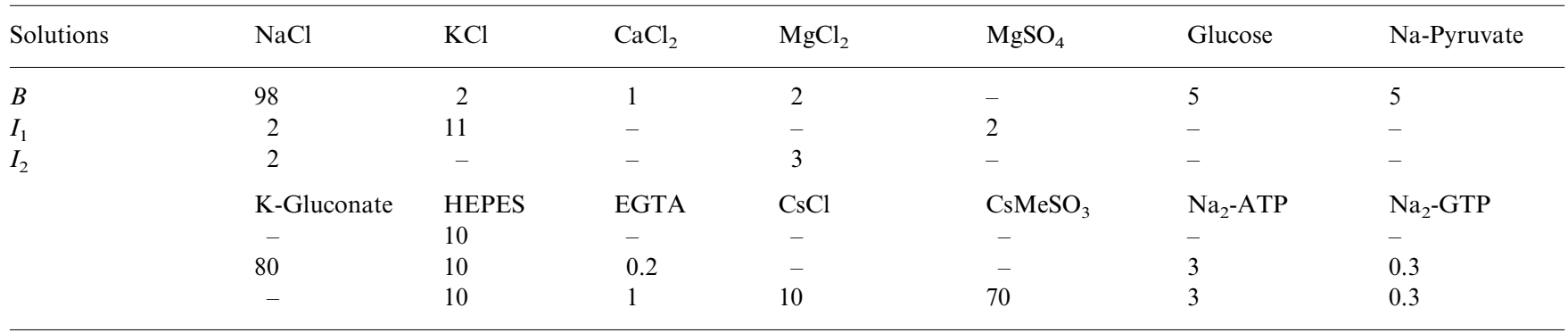

${ }^{a}$ Concentrations in $\mathrm{mM}$. 
A

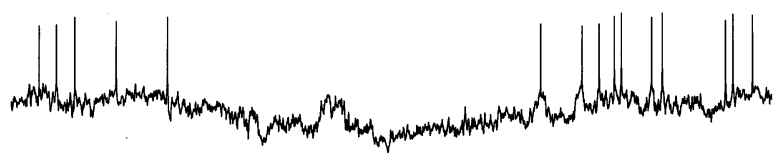

B
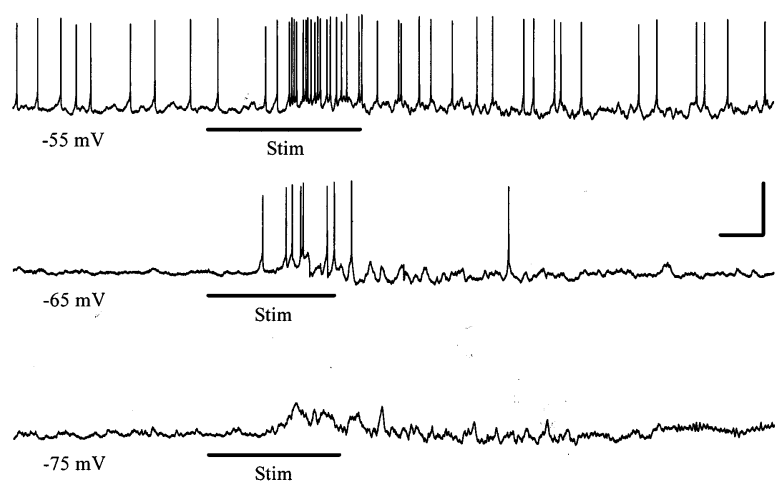

Fig. 4. Comparison between current clamp and lfVC. (A) Typical trace of membrane voltage in an olfactory receptor neuron. The fluctuation of the membrane potential do not allow reliable recordings of stimulus responses. Scale bars: $4 \mathrm{~s}, 50 \mathrm{mV}$. (B) the lfVC keeps the average membrane voltage constant. Traces are shown for reference potentials of $-75,-65$ and $-55 \mathrm{mV}$. Spontaneous activity occurred for potentials $u_{\mathrm{m}}>-65 \mathrm{mV}$. At $-75 \mathrm{mV}$ the stimulus led to a receptor potential without spiking, while at -65 and $-55 \mathrm{mV}$ a reproducible increase in spiking rate was observed. Scale bars: $2 \mathrm{~s}$, $50 \mathrm{mV}$.

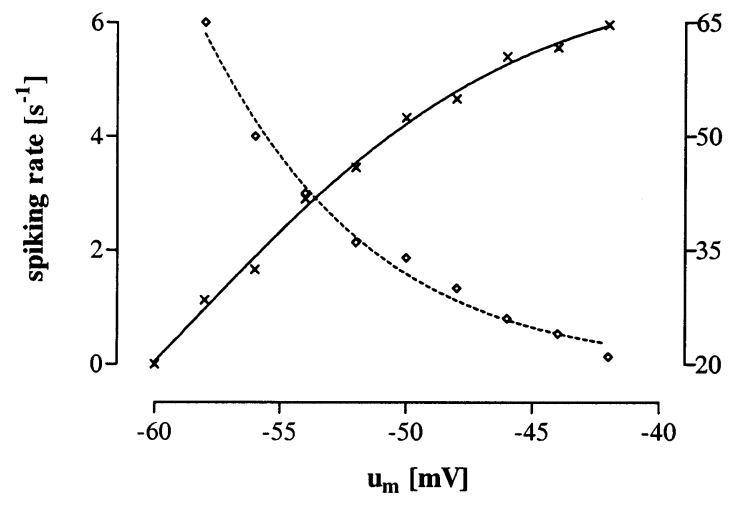

Fig. 5. Spiking rate as a function of average membrane potential in an olfactory receptor cell. In the voltage range $-60 \mathrm{mV}<u_{\mathrm{m}}<-40$ $\mathrm{mV}$, the spiking rate increased in a nonlinear way with membrane potential. The amplitude of spikes decreased with increasing average potential.

\section{Results}

We added the 1fVC to an EPC7 amplifier by: (i) picking up the $u_{\mathrm{m}}$ signal in the current clamp mode; (ii) feeding it to the custom made circuit; and (iii) inserting the signal $G\left(u_{\text {ref }}-u_{\mathrm{f}}\right)$ through $2 \mathrm{k} \Omega\left(R_{\text {inj }}\right)$ to the command summing point of the EPC7 amplifier (Fig. 1C). Other patch clamp amplifiers can be connected to the lfVC circuit in the same way. In the following we illustrate the advantages of the 1fVC by giving four examples.

1. We recorded from olfactory receptor neurons (ORNs) in the tadpole olfactory mucosa and compared the conventional current clamp configuration to the low frequency voltage clamp configuration. The voltage record in the current clamp shows considerable fluctuations with action potentials superimposed (Fig. 4A), while in the IfVC, ORNs can be held at selectable, but controlled average potentials (Fig. 4B). This facilitates reproducible recordings of odorant responses in the same cell as well as the comparison of responses in different cells.

2. One of the most straightforward applications of the IfVC is the analysis of the mean spiking rate of a neuron as a function of the average depolarization. By setting the average membrane potential of an olfactory receptor cell to a number of different values, the spiking rate increased with the average voltage as shown in Fig. 5. The concomitant decrease in spike amplitude (same figure) can most presumably be attributed to the inactivation of voltage-gated sodium channels.

3. The lfVC may also be useful when recording synaptic potentials. Fig. 6 shows three voltage traces measured in a mitral cell of the olfactory bulb. The average membrane potential was set to $-80,-40$ and $0 \mathrm{mV}$, respectively. Clearly, the synaptic potentials were depolarizing at $-80 \mathrm{mV}$, hyperpolarizing at $0 \mathrm{mV}$ and absent at $-40 \mathrm{mV}$. This coincides reasonably well with the chloride reversal potential as calculated from the composition of the solutions (Table 1).

4. Our last example shows a particular feature of mitral cells of the olfactory bulb, i.e. regenerative calcium spikes. At an average membrane potential

$\mathbf{A}$

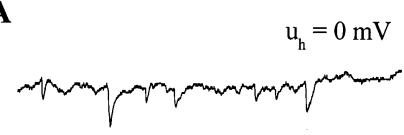

B

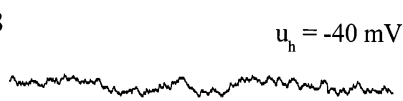

C

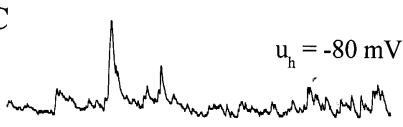

$1 \mathrm{~s} / 5 \mathrm{mV}$

Fig. 6. Postsynaptic potentials of a mitral cell of the olfactory bulb. The average holding voltage $u_{\mathrm{h}}$ is set to $0 \mathrm{mV}$ (A), $-40 \mathrm{mV}$ (B) and $-80 \mathrm{mV}(\mathrm{C})$. 
A

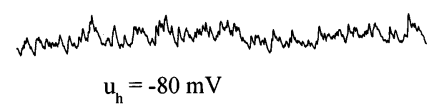

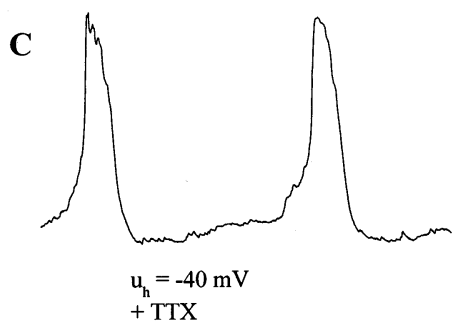

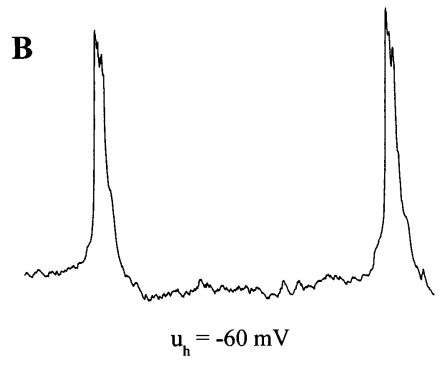

D

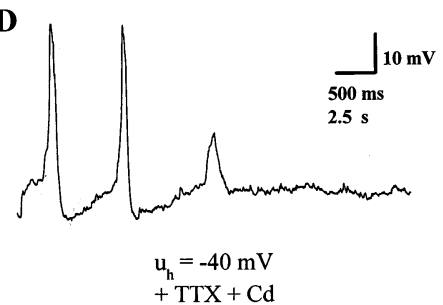

Fig. 7. Regenerative potentials in a mitral cell. (A) Control trace at an average voltage $u_{\mathrm{h}}$ of $-80 \mathrm{mV}$; (B) spikes at $-60 \mathrm{mV}$, induced by the activation of a TTX-blockable $\mathrm{Na}^{+}$-conductance; spikes disappeared during application of TTX $(1 \mu \mathrm{M}$, not shown); (C) spikes at $-40 \mathrm{mV}$ with TTX in the bath solution; (D) experimental conditions as in (C), except that $12 \mathrm{~s}$ prior to the start of the trace shown, Cd ${ }^{2+}(500 \mu \mathrm{M}) \mathrm{was}$ applied to the bath. Subsequent ceasing of the spikes during $\mathrm{Cd}^{2+}$ application. True calculation: $500 \mathrm{~ms}(\mathrm{~A}, \mathrm{~B}$ and $\mathrm{C})$ and $2.5 \mathrm{~s}(\mathrm{D})$.

$u_{\mathrm{h}}$ of $-80 \mathrm{mV}$ a control voltage trace was recorded (Fig. 7A). At an average potential of $-60 \mathrm{mV}$, regenerative potentials were observed (Fig. 7B), which disappeared after application of TTX (trace similar to Fig. 7A, not shown). When the average potential was then set to $-40 \mathrm{mV}$, regenerative potentials reappeared (Fig. 7C). As TTX was still present in the bath and as voltage gated channels are largely inactivated at $-40 \mathrm{mV}$, the regenerative potentials shown in Fig. 7C may be caused by $\mathrm{Ca}^{2+}$ currents. In fact, $\mathrm{Cd}^{2+}$ applied to the bath blocked the regenerative potentials (Fig. 7D). We conclude that the currents underlying the potentials shown in Fig. 7B were carried by sodium and by calcium ions. A detailed analysis of the calcium currents and fast prepotentials in mitral cells will be reported in a separate publication.

\section{Discussion}

In this paper we describe a method of measuring voltage transients such as action potentials or synaptic potentials, while the average voltage is kept constant. This recording configuration is similar to the current clamp in that voltages are recorded. However, owing to the particular feature that the average voltage is clamped, it is best characterized as a low frequency voltage clamp.

We designed the IfVC as an external device that can be attached to any patch clamp amplifier. Except for the current injection, the internal circuitry of the patch clamp amplifier remains untouched. This has the advantage that the IfVC is independent of the specific patch clamp amplifier used. It just uses the current clamp mode in a particular way. In a future development, one could consider increasing the frequency discrimination of the circuitry by implementing an 8-pole lowpass filter rather than a 1-pole filter in the feedback loop.

The lfVC can be used in situations where the current clamp usually would be used, except for the measurement of the steady state membrane potential. It can also be useful under circumstances that are unfavourable for voltage clamp measurements, e.g. in perforated patch recordings with high access resistances and long voltage clamp time constants. We found the IfVC particularly useful in the study of olfactory receptor neurons, where we increased the endogenously very low spiking rate to a moderate level prior to stimulus application (Vogler and Schild, 1999).

It is interesting to look at the lfVC as an artificial membrane conductance. As the circuit injects the current $\left(\left(u_{\text {ref }}-u_{\mathrm{f}}\right) G-u_{\mathrm{m}}\right) / R_{\text {inj }}$ onto the membrane capacitance and as the membrane capacitor of a cell does not distinguish currents entering through the pipette from currents crossing the plasma membrane, the lfVC has the effect of an additional membrane conductance. This is seen in the experiment shown in Fig. 7, in which $\mathrm{K}^{+}$ channels were blocked by $\mathrm{Cs}^{+}$added to the pipette solution. The potential at which the current through the pipette was $I=0$, was $\approx 20 \mathrm{mV}$ in this experiment. The repolarization of the regenerative potentials was brought about by a hyperpolarizing, slowly activating 
current injected by the lfVC rather than by a hyperpolarizing membrane conductance. A detailed study of the regenerative potentials in mitral cells of the olfactory bulb will be reported in the near future.

The full electronic schematics of the lfVC, includes lowpass filtering of the reference voltage $\left(\tau_{\text {ref }}=4.4 \mathrm{~s}\right)$ in order to prevent large excursions of the membrane voltage, and a number of analog switches and timers to guarantee that the switch from the conventional 'current clamp and command' mode to the IfVC mode occurs sufficiently slowly. An abrupt switching could, depending on the actual membrane potential and the setting of $u_{\text {ref }}$, destroy the cell by injection of too large a current.

\section{Note added in proof}

The 'mixed clamp' device (Gebhardt and Lindemann, 1970; Gebhardt, 1974) designed for clamping epithelial membranes had some functional similarity with the IfVC. The electronics were, however, quite different.

\section{Acknowledgements}

We thank the experts of our electronic workshop, Mr. Klugmann and Mr. Werner, for their excellent collabo- ration and for building the prototypes of the low frequency voltage clamp device.

\section{References}

Gebhardt U, Lindemann B. Fast switching between voltage- and current control of membrane clamps. Med Biol Eng 1970;8:3157.

Gebhardt U. A fast voltage clamp with automatic compensation for changes of extracellular resistivity. Pflügers Archiv 1974;347:1-7.

Hamill OP, Marty A, Neher E, Sakmann B, Sigworth FJ. Improved patch-clamp techniques for high-resolution current recording from cells and cell-free membrane patches. Pflügers Arch 1981;391:85-100.

Nezlin LP, Schild D. Structure of the olfactory bulb in tadpoles of Xenopus laevis, Cell Tiss Res 2000 (in press).

Nieuwkoop PD, Faber J. Normal table of Xenopus laevis (Daudin). Amsterdam, Holland: North Holland, 1956.

Ogata K. Modern Control Engineering. Upper Saddle River: Prentice Hall, 1996.

Schild D. A computer-controlled device for the application of odours to aquatic animals. J Electrophysiol Techn 1985;12:71-9.

Schild D, Gennerich A, Schultens HA. Microcontrollers as inexpensive pulse generators and parallel processors in electrophysiological experiments. Med Biol Eng Comput 1996;34:305-7.

Vogler C, Schild D. Inhibitory and excitatory responses of olfactory receptor neurons of Xenopus laevis tadpoles to stimulation with amino acids. J Exp Biol 1999;202:997-1003. 